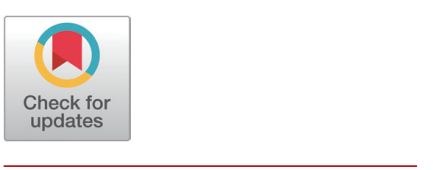

* These authors contributed equally to this work.

Received: September 7, 2021

Revised: October 25, 2021

Accepted: November 17, 2021

Corresponding author

Youngmi Jung

Dept. of Integrated Biological Science,

Pusan National University, Busan 46241,

Korea.

Tel: +82-51-510-2262

E-mail:y.jung@pusan.ac.kr

Copyright $\odot 2021$ The Korean Society of Developmental Biology.

This is an Open Access article distributed under the terms of the Creative Commons Attribution Non-Commercial License (http://creativecommons.org/licenses/ by-nc/4.0/) which permits unrestricted non-commercial use, distribution, and reproduction in any medium, provided the original work is properly cited.

ORCID

Jinsol Han

https://orcid.org/0000-0002-5317-9409 Chanbin Lee

https://orcid.org/0000-0002-5680-9613

Youngmi Jung

https://orcid.org/0000-0003-2189-0658

Conflict of interests

The authors declare no potential conflict of interest.

Acknowledgements

This work was supported by a 2-Year Research Grant of Pusan National

University to Y.J and BK21 Four Program of

Pusan National University to C.L.

\section{Deficiency of Formyl Peptide Receptor 2 Retards Hair Regeneration by Modulating the Activation of Hair Follicle Stem Cells and Dermal Papilla Cells in Mice}

\author{
Jinsol Han ${ }^{1, *}$, Chanbin Lee ${ }^{1, *}$, and ${ }^{\dagger}$ Youngmi Jung ${ }^{1,2}$ \\ ${ }^{1}$ Dept. of Integrated Biological Science, Pusan National University, Busan 46241, Korea \\ ${ }^{2}$ Dept. of Biological Sciences, Pusan National University, Busan 46241, Korea
}

\section{Abstract}

Hair loss is one of the most common chronic diseases, with a detrimental effect on a patient's psychosocial life. Hair loss results from damage to the hair follicle (HF) and/or hair regeneration cycle. Various damaging factors, such as hereditary, inflammation, and aging, impair hair regeneration by inhibiting the activation of hair follicle stem cells (HFSCs) and dermal papilla cells (DPCs). Formyl peptide receptor 2 (FPR2) regulates the inflammatory response and the activity of various types of stem cells, and has recently been reported to have a protective effect on hair loss. Given that stem cell activity is the driving force for hair regeneration, we hypothesized that FPR2 influences hair regeneration by mediating HFSC activity. To prove this hypothesis, we investigated the role of FPR2 in hair regeneration using Fpr2 knockout (KO) mice. Fpr2 KO mice were found to have excessive hair loss and abnormal HF structures and skin layer construction compared to wild-type (WT) mice. The levels of Sonic hedgehog (Shh) and $\beta$-catenin, which promote HF regeneration, were significantly decreased, and the expression of bone morphogenetic protein (Bmp)2/4, an inhibitor of the anagen phase, was significantly increased in Fpr2 KO mice compared to WT mice. The proliferation of HFSCs and DPCs was significantly lower in Fpr2 KO mice than in WT mice. These findings demonstrate that FPR2 impacts signaling molecules that regulate HF regeneration, and is involved in the proliferation of HFSCs and DPCs, exerting a protective effect on hair loss.

Keywords: Formyl peptide receptor 2, Hair follicle, Hair loss, Hair regeneration, Skin

\section{INTRODUCTION}

Hair is a major skin appendage that plays an important role in protection, thermoregulation, and sensory function (Stenn \& Paus, 2001). However, various damaging factors, such as aging, hereditary, inflammation, and physiological stress, induce excessive hair loss regardless of gender or age (Strazzulla et al., 2018). Given that hair deeply influences an individual's appearance, unwanted hair loss can result 
Authors' contributions

Conceptualization: Jung Y.

Data curation: Jung Y.

Formal analysis: Han J, Lee C, Jung Y

Methodology: Han J, Lee C, Jung Y.

Software: Han J, Lee C.

Validation: Han J, Lee C, Jung Y.

Investigation: Han J, Lee C.

Writing-original draft: Han J, Jung Y.

Writing-review \& editing: Han J, Lee C, Jung Y.

Ethics approval

All animal care was carried out according to

the provisions of the National Institutes of

Health (NIH) guidelines for the care and Use of

Laboratory. The animal protocol used in this

study has been approved by the Pusan National

University-Institutional Animal Care and Use

Committee (PNU-IACUC: PNU-2020-2574) on

their ethical procedures and scientific care. in lower self-esteem and quality of life, leading to psychological problems (Grimalt, 2005). Many studies have been conducted on hair loss and treatments, and two Food and Drug Administration (FDA)-approved drugs have been developed: minoxidil and finasteride (Lee et al., 2018; Suchonwanit et al., 2019). However, the effects of the drugs vary from person to person, and there is often a variety of side effects (Rossi et al., 2012; Carreño-Orellana et al., 2016). In particular, finasteride is applied only to male patients as a treatment for male-pattern hair loss (Seale et al., 2016). Hence, developing effective therapeutics for all types of hair loss is an urgent task.

Hair grows up from the hair follicle (HF), a complex mini-organ that includes nerves, blood vessels, sebaceous glands, and arrector pili muscles (Schneider et al., 2009). After embryonic HF morphogenesis, the HF repeats a cycle consisting of the anagen, catagen, and telogen phases to regenerate hair for life (Stenn \& Paus, 2001; Schneider et al., 2009). Anagen is referred to as an active growth phase because follicular cells actively proliferate and remodel the HF structure for maturation and pigmentation of hair fibers (Schneider et al., 2009). Mature HFs begin to regress as they move into the catagen phase, which features an increase in apoptosis (Schneider et al., 2009). Overall HF length is substantially reduced during the catagen phase, and the HF proceeds to the telogen, or resting phase (Schneider et al., 2009). In the telogen phase, HFs are inactivated and have minimal length (Müller-Röver et al., 2001). Cyclic conversion to the anagen phase follows the telogen phase (Chen et al., 2020a). Hence, for normal hair growth after embryonic HF morphogenesis, the transition from the telogen to the anagen phase is a key step for hair regeneration (Chen et al., 2020a).

The transition is controlled by the delicate balance of signaling molecules in HFs, which mediate the activation of HF stem cells (HFSCs) and mesenchymal dermal papilla cells (DPCs) (Chen et al., 2020a). Several signaling molecules, such as Sonic hedgehog (Shh), Wnt/ $\beta$-catenin, and bone morphogenetic protein (BMP) $2 / 4$, have been shown to be involved in hair regeneration by regulating the activities of HFSCs and DPCs (Rishikaysh et al., 2014). Shh and Wnt/ $\beta$-catenin signaling induce the proliferation of HFSCs and maintain the differentiation potential of DPCs to hair, promoting hair regeneration (Rishikaysh et al., 2014; Avigad Laron et al., 2018). While BMP2 and BMP4 signaling results in inactivation of HFSCs and prevention of them from progressing to HF regeneration by holding HFs in a refractory telogen phase (Botchkarev et al., 2002; Zhang et al., 2006). However, the crosstalk between these stem cells via signaling molecules is complex and poorly understood. Even though several signaling molecules regulating the hair regeneration activities of HFSCs and DPCs have been studied, it remains unclear how the activation of these molecules is triggered. In addition, skin is a complex organ in which more than 50 cell types contribute to skin structures (Abaci et al., 2018); hence, the effects of the various cells surrounding HFs on stem cells are not fully proven.

Formyl peptide receptor 2 (FPR2), which belongs to the G protein-coupled receptor family, is an important regulator of inflammation (Chen et al., 2020b). Recent studies have investigated the expression and role of FPR2 in nonphagocytic cells, and several have reported that various types of stem cells and dermal fibroblasts express FPR2, and that activation of FPR2 promotes migration and proliferation of stem cells, such as neural stem cells and dental stem cells (VanCompernolle et al., 2003; Zhang et al., 2017; Gaudin et al., 2018). Tsuruki et al. showed that FPR2 prevented hair loss in rats that received a chemotherapy drug (Tsuruki \& Yoshikawa, 2006). FPR2 agonists, such as N-formyl-Met-Leu-Phe (fMLP) and MMK-1, suppressed hair loss by activating nuclear factor kappa-light-chain-enhancer of activated B cells (NF- $\kappa$ B) signaling, which blocked apoptosis (Tsuruki \& Yoshikawa, 2006). It was proved that NF- $\kappa \mathrm{B}$ activated by the FPR2 agonist was necessary for HFSC proliferation and activation for the induction of the anagen phase (Krieger et al., 2018). Krieger et al. also reported that NF- $\kappa$ B activity was detected in anagen induction, and 
mice with HF-specific inactivation of NF- $\kappa \mathrm{B}$ had a delayed transition of telogen to anagen phase compared to control mice (Krieger et al., 2018). Given that FPR2 is expressed in various types of stem cells and its protective role in hair loss, we hypothesized that FPR2 might be involved in the $\mathrm{HF}$ cycle and impact hair regeneration. We found that Fpr2 knockout (KO) mice experienced extensive hair loss in the dorsal skin 18 weeks after birth, and that the hair loss was caused by Fpr2 deficiency-induced inactivation of HFSCs and DPCs. These findings suggest the therapeutic potential of FPR2 in the treatment of hair loss.

\section{MATERIALS AND METHODS}

\section{Animals}

Male C57BL/6 mice (6-week-old) were purchased from Hyochang (Daegu, Korea) and used for wild-type (WT) controls. Male Fpr2 KO mice were generously donated from Dr. Kim (Pusan National University School of Medicine, Yangsan, Korea). Mice were housed with 12-hour light/ dark cycle with free access to normal chow diet and water. 18-week-old mice were sacrificed to obtain skin sample. All animal care was carried out according to the provisions of the National Institutes of Health (NIH) guidelines for the care and Use of Laboratory. The animal protocol used in this study has been approved by the Pusan National University-Institutional Animal Care and Use Committee (PNU-IACUC) on their ethical procedures and scientific care (Approval Number PNU-2020-2574).

\section{Western blotting}

Total protein was extracted from dorsal skin tissues that had been stored at $-80^{\circ} \mathrm{C}$. Samples were cryo-pulverized and the tissue powder was mixed in triton lysis buffer (TLB) supplemented with protease inhibitors (Complete mini; Roche, Indianapolis, IN, USA) and centrifuged at 13,000 r.c.f. for 15 minutes at $4^{\circ} \mathrm{C}$. The supernatants containing protein extracts were used in subsequent biochemical analysis. Protein concentration was measured by Pierce BCA Protein Assay Kit (Thermo Fisher Scientific, Waltham, MA, USA). To denature and reduce protein samples, proteins were boiled in $5 \mathrm{X}$ sample buffer containing $\beta$-mercaptoethanol and sodium dodecyl sulfate (SDS) at $100^{\circ} \mathrm{C}$ for 10 minutes. Total $70 \mu \mathrm{g}$ of protein lysates was separated by SDS-polyacrylamide gel electrophoresis (PAGE) on 10\% tris-glycine gel and transferred onto a $0.45 \mu \mathrm{m}$ pore size polyvinylidene difluoride (PVDF) membrane (Millipore, Darmstadt, Germany). Primary antibodies used in this study were as follows: rabbit anti-FPR2 antibody (diluted 1:100; Novus Biologicals, Centennial, CO, USA), rabbit anti-Shh antibody (diluted 1:100; Santa Cruz Biotechnology, Dallas, TX, USA), rabbit anti-nonphospho $\beta$-catenin antibody (diluted 1:1,000; Cell Signaling Technology, Danvers, MA, USA), rabbit anti-BMP4 antibody (diluted 1:1,000; R\&D Systems, Minneapolis, MN, USA), rabbit anti-BMP2 antibody (1:1,000; Bioss, Woburn, MA, USA), mouse anti-Keratin 15 antibody (1:1,000; Thermo Fisher Scientific), rabbit antiCD133 (1:1,000; abcam, Cambridge, MA, USA) and mouse anti-glyceraldehydes 3-phosphate dehydrogenase antibody (GAPDH) (diluted 1:1,000; Bio-rad, Hercules, CA, USA) were used in this experiment. Horseradish peroxidase-conjugated anti-rabbit or anti-mouse IgG (Enzo Life Sciences, Farmingdale, NY, USA) was used as secondary antibody. Protein bands were detected using an EzWestLumi ECL solution (ATTO, Tokyo, Japan) as per the manufacturer's specifications (ATTO, Ez-Capture II). Densities of protein bands were measured using CS Analyzer software (Version1.0.3, ATTO). 


\section{Skin histology}

Skin specimens were fixed in 10\% neutral buffered formalin (Sigma-Aldrich), embedded in paraffin and cut into $6-\mu \mathrm{m}$-thick sections. Sections were deparaffinized, hydrated, and stained in hematoxylin (Gill's Hematoxylin V, Muto Pure Chemicals, Tokyo, Japan) for 15 min at room temperature. And then stained in eosin (1\% Eosin Y Solution, Muto Pure Chemicals) for 5 min at room temperature. Slides were viewed with an Olympus CX41 light microscope (Olympus Optical, Tokyo, Japan) and morphometric analysis of stained regions in the tissue sections was performed using cellSens software (Olympus Optical).

\section{Immunofluorescence staining}

For immunofluorescence staining, skin tissues were embedded in OCT $\left(\right.$ Scigen $^{\mathrm{TM}}$, Paramount, CA, USA) and immediately frozen on dry ice. Frozen blocks were cut into $10 \mu \mathrm{m}$ using a cryostat (Leica 3050s, Leica Biosystems, High Peak, UK), and tissue sections were thaw mounted onto glass slides. Slides were fixed in and permeabilized with acetone and methanol, respectively. They were washed with tris-buffered saline (TBS) and incubated with blocking solution (Dako, CA, USA) for 30 minutes. Sections were incubated with primary antibody, rabbit anti-FPR2 antibody (diluted 1:1,000; Novus Biologicals), rabbit anti-Shh antibody (diluted 1:150; SantaCruz, Dallas, TX, USA), rabbit anti- $\beta$-catenin antibody (diluted 1:800; Cell Signaling Technology), rabbit anti-BMP4 antibody (diluted 1:100; R\&D Systems), rabbit anti-BMP2 antibody (diluted 1:500; Bioss), mouse anti-Keratin 15 antibody (diluted 1:500; Thermo Fisher Science), rabbit-anti CD133 antibody (diluted 1:100; abcam) for $4^{\circ} \mathrm{C}$ overnight. Slides were washed in TBS and incubated with fluorescein labelled secondary antibody, Alexa Fluor 568 anti-rabbit IgG (Invitrogen, Carlsbad, CA, USA) and Alexa Fluor 488 anti-mouse $\mathrm{IgG}$ (Invitrogen) for $30 \mathrm{~min}$ at room temperature. For double immunofluorescence staining, slides were removed from secondary antibody solution and washed with TBS. Slides were incubated with blocking solution for 10 minutes, then with secondary primary antibody, $\alpha$-SMA (diluted 1:500; abcam) for 2 hours at room temperature. Slides were washed in TBS and incubated with fluorescein-labelled secondary antibody, Alexa Fluor 488 anti-mouse IgG (Invitrogen) for 30 minutes at room temperature. Slides were mounted on slides antifade mounting medium with 4',6-diamidno-2-phenylinole (DAPI, VectaShield, Burlingame, CA, USA). Slides were viewed with a Leica DMi8 microscope (Leica Microsystems, Wetzlar, Germany).

\section{Statistics}

Results are expressed as the mean \pm SEM. Statistical differences were analyzed by unpaired twosample student's $t$-test. $p$-values $<0.05$ were considered as statistically significant.

\section{RESULTS}

\section{Fpr2 KO mice display excessive hair loss in the dorsal skin}

Although WT and Fpr2 KO mice had normal hair growth after birth, severe hair loss in the dorsal skin was observed in Fpr2 $\mathrm{KO}$ mice with an average age of 18 weeks, but not in WT mice (Fig. 1A). To examine histological differences in the dorsal skin of these mice, we conducted the $\mathrm{H} \& \mathrm{E}$ staining. The skin structure of the WT mice showed a thin hypodermis adipocyte layer and evenly distributed HFs in the dermis (Fig. 1B). All HFs were upright toward the epidermis, and appendages such as sebaceous glands also had normal structure. However, the skin structure of the Fpr2 KO mice was different from that of the WT mice. The hypodermis adipocyte layer was 
A

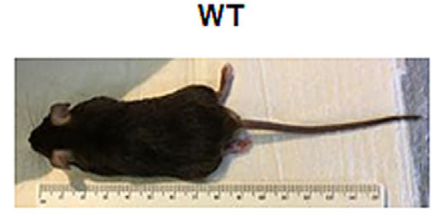

Fpr2 KO

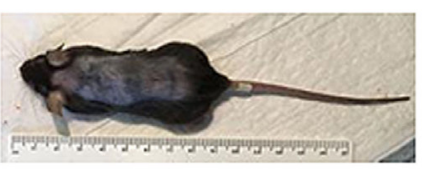

B

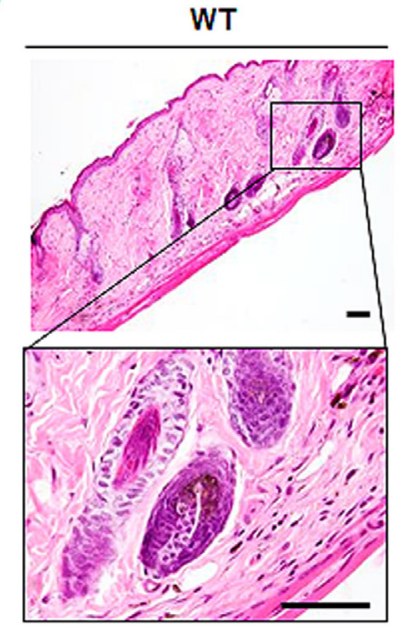

Fpr2 KO

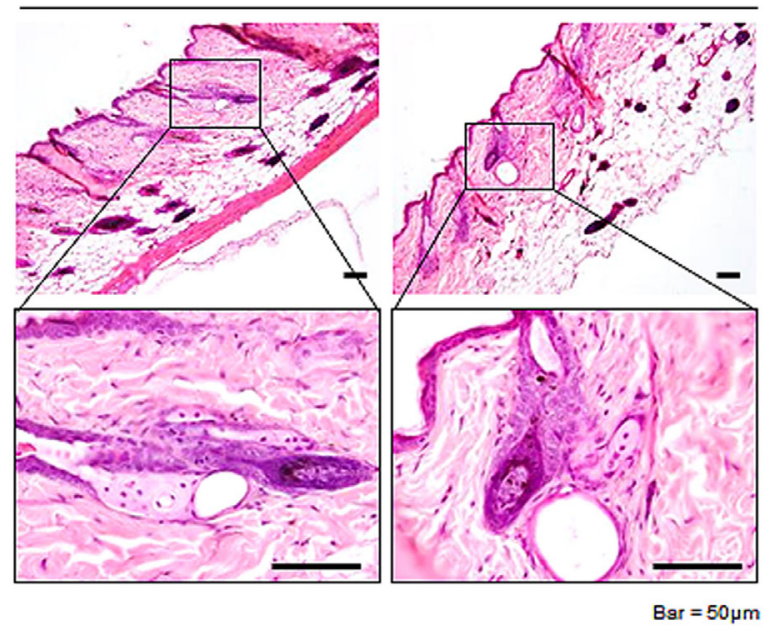

Fig. 1. Fpr2 KO mice exhibit hair loss in the dorsal skin of the body. (A) Macroscopic images of the dorsal area of representative WT and Fpr2 KO mice. (B) Representative images of H\&E-stained dorsal skin sections from these mice $(\times 10)$. Magnified images $(\times 40)$ are shown in bottom panel (Scale bars: $50 \mu \mathrm{m})$.

much thicker in $\mathrm{KO}$ mice than in WT mice, and HFs were clustered or irregularly distributed in $\mathrm{KO}$ mice. Moreover, the HF structure was abnormal, presenting a bent and cyst-like structure. Sebaceous glands also had cyst-like structures in the $\mathrm{KO}$ mice. The distorted structure of the dorsal skin in the $\mathrm{KO}$ mice indicated that $\mathrm{Fpr} 2$ deletion might be involved in generating abnormal HFs.

\section{Fpr2 is expressed in hair follicles}

Before investigating the role of Fpr2 in the skin, we first assessed Fpr2 expression in dorsal skin tissue. Using western blot analysis, we found that WT mice had a significantly increased expression of Fpr2, whereas Fpr2 KO mice rarely expressed Fpr2 in the dorsal skin (Fig. 2A, B). In line with quantification data, Fpr2-expressing cells were evident in the outer layer of the lower $\mathrm{HF}$ and scattered in the dermis (indicated by arrows) of the WT mice (Fig. 2C). However, these cells were hardly detected in the Fpr2 $\mathrm{KO}$ mice. To identify the cells that were positive for Fpr2, we conducted double immunofluorescence staining for Fpr2 and $\alpha$-Smooth muscle actin ( $\alpha$-Sma), a well-known fibroblast marker (Fang et al., 2013). Because FPR2 is reportedly expressed in dermal fibroblasts (VanCompernolle et al., 2003), we expected to detect Fpr2 expression in these cells. Expression of $\alpha$-Sma colocalized with Fpr2-positive cells in the outer layer of HFs in WT mice (Fig. 2D). These double-positive cells were not observed in the Fpr2 KO mice. These results demonstrate that Fpr2 is expressed by dermal fibroblasts located in the outer layer of the HF and dermis. 
A

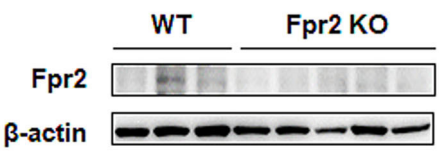

B

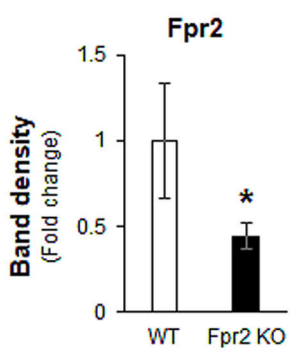

C
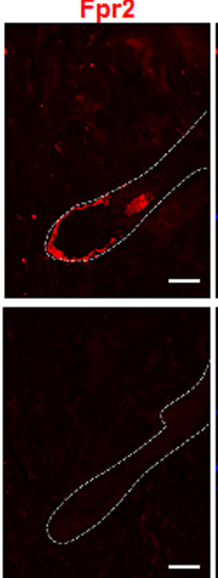

.
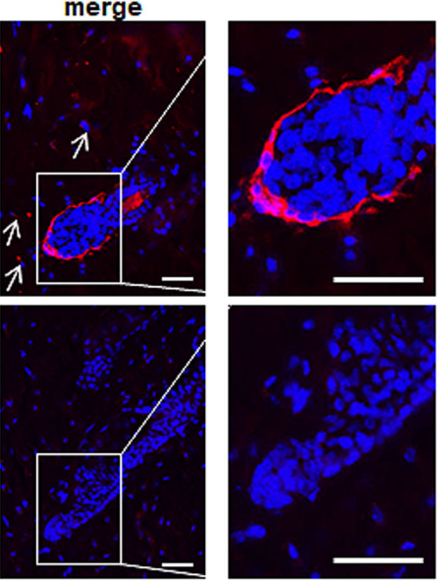

$\mathrm{Bar}=20 \mu \mathrm{m}$
D

WT
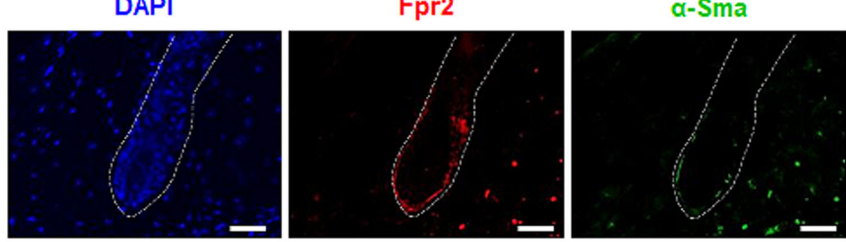
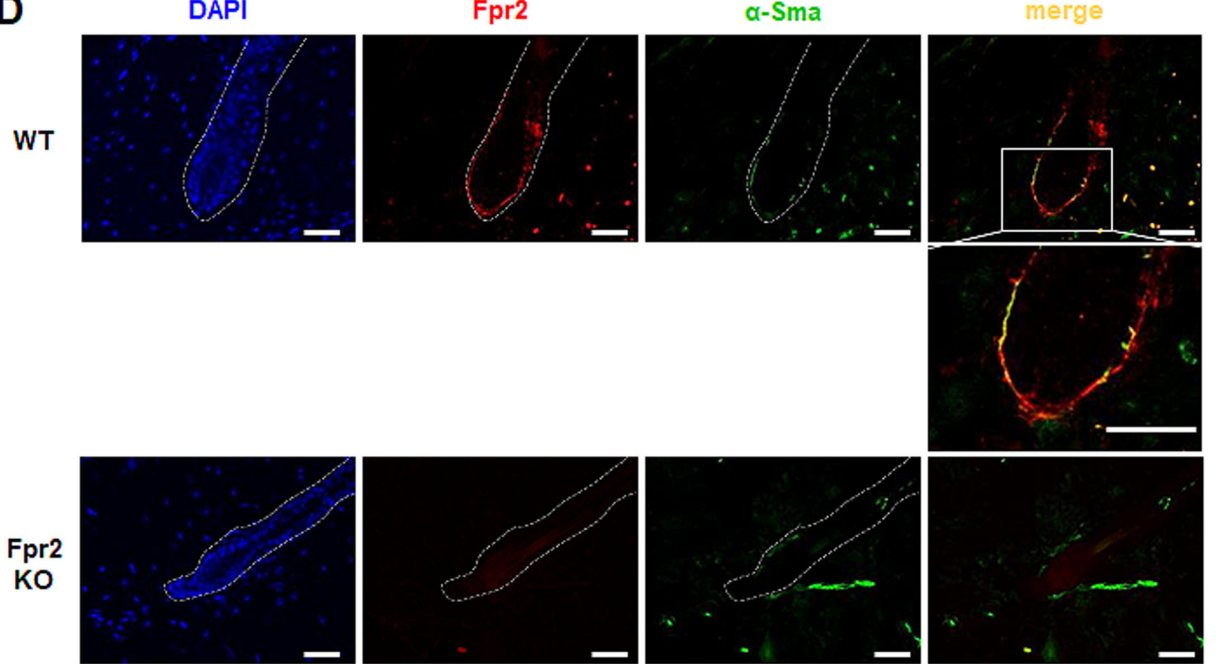

Fig. 2. Expression of Fpr2 in dorsal skin of mice. (A) Western blot analysis and (B) cumulative densitometric analysis for Fpr2 in dorsal skin of WT and Fpr2 KO mice. Band densities were normalized to the expression level of $\beta$-actin, an internal control. Data represent the mean $\pm S E M$ (unpaired two-sample student's $t$-test; $n \geq 3$ /group, * $p<0.05$ vs WT). (C) Representative images of immunofluorescence staining for Fpr2 in the dorsal skin sections from these mice $(\times 40)$. Magnified images $(\times 100)$ are shown in the right panel. DAPI was used in staining for nucleus. Arrow indicates the scattered cells expressing Fpr2 in dermis (Scale bars: $20 \mu \mathrm{m}$ ). (D) Double immunofluorescence staining images for Fpr2 (red) with $\alpha$-Sma (green) in the dorsal skin sections from these mice $(\times 40)$. DAPI (blue) was used as nuclear counterstaining. The white dashed lines indicate the HF regions. Magnified images $(\times 100)$ are shown in under panel of merged image (Scale bars: $20 \mu \mathrm{m}$ ). $\alpha$-Sma, $\alpha$-smooth muscle actin.

\section{Fpr2 suppression downregulates inducers of hair regeneration (Shh and $\beta$-catenin) and upregulates inhibitors of hair regeneration (Bmp2 and Bmp4)}

After HF morphogenesis, HFs undergo cycles of growth (anagen), regression (catagen), and rest (telogen) to produce new hairs for life (Schneider et al., 2009). The progression of the hair regeneration cycle is modulated by several signaling factors, such as $\mathrm{SHH}, \mathrm{Wnt} / \beta$-catenin and BMPs (Botchkarev et al., 2002; Zhang et al., 2006; Rishikaysh et al., 2014; Avigad Laron et al., 
2018). Expression of Shh in adjacent DPCs and activation of $\mathrm{Wnt} / \beta$-catenin signaling in HFs are necessary in the transition from the telogen to the anagen phase during hair regeneration (StJacques et al., 1998; Chiang et al., 1999; Cui et al., 2011). Deletion of these signaling molecules has been shown to result in dramatic hair shortening and/or loss (Cui et al., 2011). Given that Fpr2 KO mice showed hair loss after normal morphogenesis, we hypothesized that a lack of Fpr2 induced an alteration in these signaling molecules, and dysregulated the hair regeneration cycle. To prove our hypothesis, we evaluated Shh and $\beta$-catenin expression in the dorsal skin, including the HFs. The levels of Shh and $\beta$-catenin in the skin were significantly lower in the Fpr2 KO mice than in the WT mice (Fig. 3A, B). Shh (red colored) was expressed in DPC-like cells in the lower part of the HFs of the WT mice, whereas these cells were rarely detected in the Fpr2 KO mice, as assessed by immunofluorescence staining (Fig. 3C, left panel). Nuclear $\beta$-catenin, which indicates the activation of Wnt signaling, was apparent in the WT mice. However, in the Fpr2 KO mice, $\beta$-catenin was restricted to the cytoplasm (Fig. 3C, right panel).

In the regulation of the hair regeneration cycle, BMP2 and BMP4 are critical, as they inhibit hair regeneration by counteracting Shh and Wnt/ $\beta$-catenin (Genander et al., 2014). Their expression levels were low in the anagen phase and high in the telogen phase (Plikus et al., 2009). In the Fpr2 $\mathrm{KO}$ mice, Bmp2 and Bmp4 were significantly elevated compared to levels in the

A

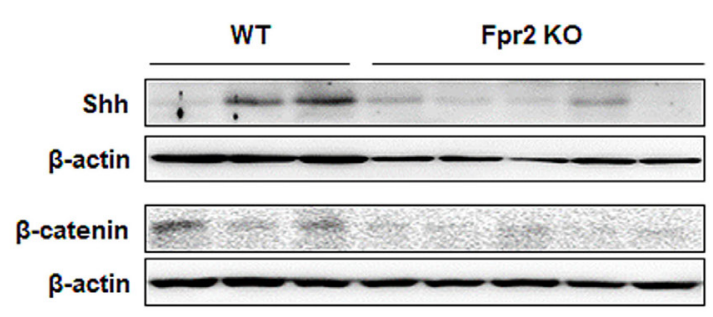

B

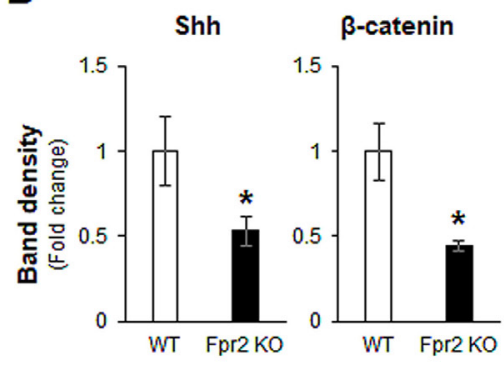

C
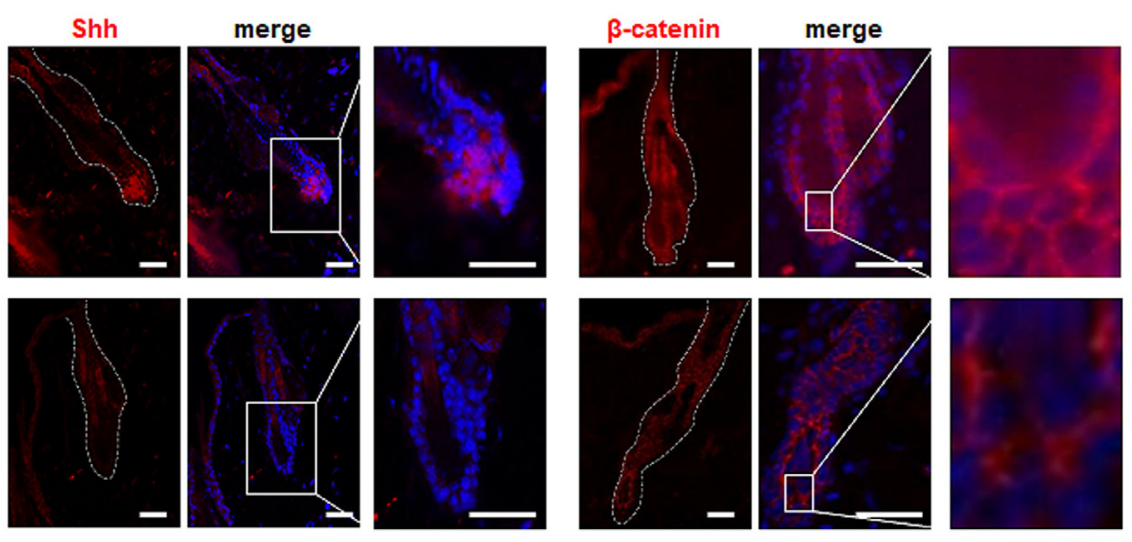

Bsr $=20 \mu \mathrm{m}$

Fig. 3. Deficient Fpr2 alleviates expression of Shh and $\beta$-catenin in dorsal skin of mice. (A) Western blot analysis and (B) cumulative densitometric analysis for Shh and $\beta$-catenin in dorsal skin of WT and Fpr2 KO mice. Band densities were normalized to the expression level of $\beta$-actin, an internal control. Data represent the mean \pm SEM (unpaired two-sample student's $t$-test; $n \geq 3$ /group, * $p<0.05$ vs WT). (C) Representative images of immunofluorescence staining for Shh (red) and $\beta$-catenin (red) in the dorsal skin sections from these mice $(\times 40)$. Magnified images $(\times 100)$ for Shh are shown in left panel and for $\beta$-catenin are shown in middle panel. DAPI was used in staining for nucleus. The white dashed lines indicate the HF regions (Scale bars: $20 \mu \mathrm{m}$ ). Shh, Sonic hedgehog. 
WT mice (Fig. 4A, B). Furthermore, Bmp2-positive cells were more obviously observed in the immunofluorescence staining of the Fpr2 $\mathrm{KO}$ mice, whereas these cells were hardly detected in the WT mice (Fig. 4C, left panel). In addition, Bmp4-expressing cells were more evident in the Fpr2 KO mice than in the WT mice, and these cells were observed in fibroblast-like cells located in the extrafollicular dermis (Fig. 4C, right panel). Taken together, these findings suggest that Fpr2 ablation arrests the hair regeneration cycle in the telogen phase by decreasing Shh and $\beta$-catenin and increasing Bmp2/4 expression.

\section{Deletion of Fpr2 decreases activation of hair follicle stem cells (HFSCs) and dermal papilla cells (DPCs)}

During hair regeneration, HFSCs proliferate and differentiate into HF components, and DPCs, which are now considered a reservoir of multipotent stem cells, regulate HF development and growth (Rahmani et al., 2014; Ji et al., 2021). HFSCs and DPCs undergo rapid proliferation to fuel the initial stage of hair growth that supports the prolonged growth of the hair (Rahmani et al., 2014; Ji et al., 2021). Based on the inhibited hair regeneration observed in the Fpr2 KO mice, we examined the activation of HFSCs and DPCs by assessing the specific activation markers for each cell type.

A

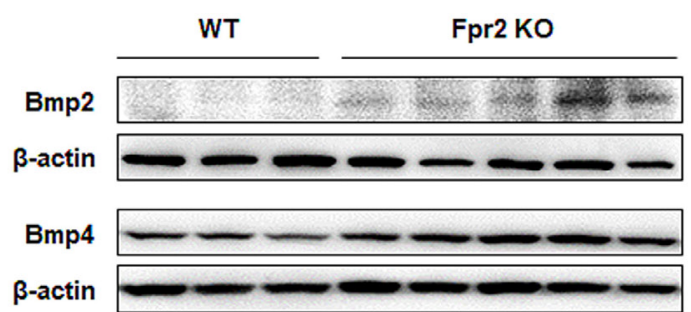

C
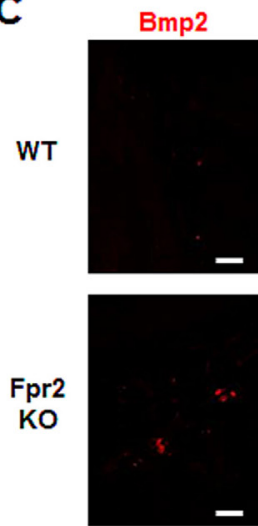

merge
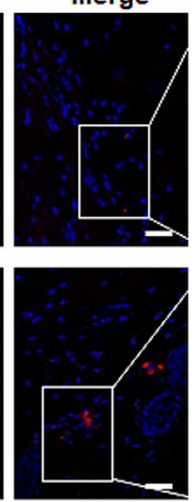
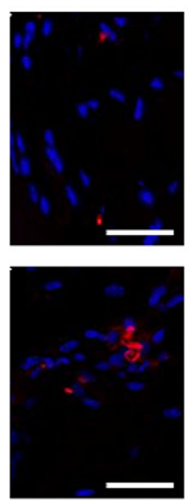

B

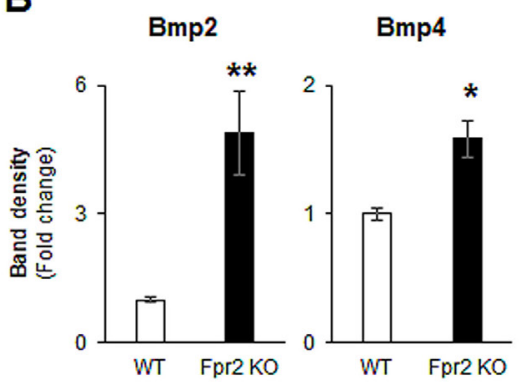

Bmp4
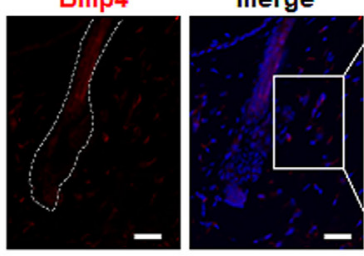

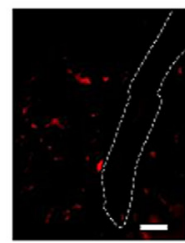

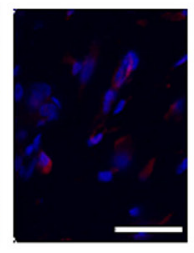

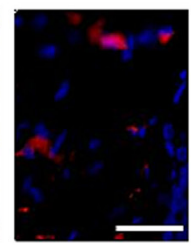

Bar $=20 \mu \mathrm{m}$

Fig. 4. Levels of Bmp2 and Bmp4 are elevated in dorsal skin of Fpr2 KO mice. (A) Western blot analysis and (B) cumulative densitometric analysis for Bmp2 and Bmp4 in dorsal skin of WT and Fpr2 KO mice. Band densities were normalized to the expression level of $\beta$-actin, an internal control. Data represent the mean \pm SEM (unpaired two-sample student's $t$-test; $\mathrm{n} \geq 3$ /group, ${ }^{*} p<0.05$, ${ }^{* *} p<0.005$ vs WT). (C) Representative images of immunofluorescence staining for Bmp2 (red) and Bmp4 (red) in the dorsal skin sections from these mice $(\times 40)$. Magnified images $(\times 100)$ are shown in right panel. DAPI was used in staining for nucleus. The white dashed lines indicate the HF regions (Scale bars: $20 \mu \mathrm{m})$. BMP, bone morphogenetic protein. 
Keratin 15 (K15) is a stem cell marker of HFs, and K15-positive HFSCs preferentially proliferate during the anagen phase (Bose et al., 2013). The level of K15 was significantly downregulated in the Fpr2 KO mice compared with the WT mice (Fig. 5A, B). Immunofluorescence staining for K15 also showed that K15-positive HFSCs were present in the WT mice, but absent from the Fpr2 KO mice (Fig. 5C, left panel).

CD133 is expressed by a subpopulation of DPCs during the early stages of the anagen phase (Ito et al., 2007). Expression of CD133 was significantly lower in the dorsal skin tissue of Fpr2 KO mice than in WT mice (Fig. 5A, B). WT mice were found to have CD133-expressing DPC-like cells located in the lowest part of the HF, whereas these cells were not detected in Fpr2 $\mathrm{KO}$ mice (Fig. 5C, right panel). These data indicate that FPR2 is involved in the proliferation of HFSCs and DPCs, thereby impacting hair regeneration.

\section{DISCUSSION}

Hair loss occurs across all ages, sexes, and ethnicities (Strazzulla et al., 2018). There are no radical therapies that prevent or reverse hair loss. Despite many studies being conducted to elucidate

A

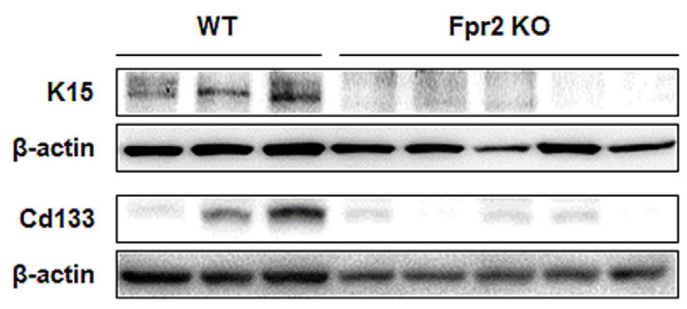

B
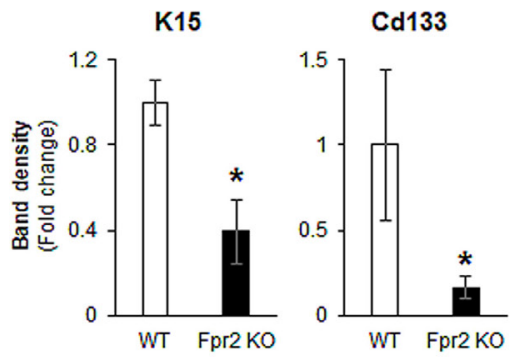

C
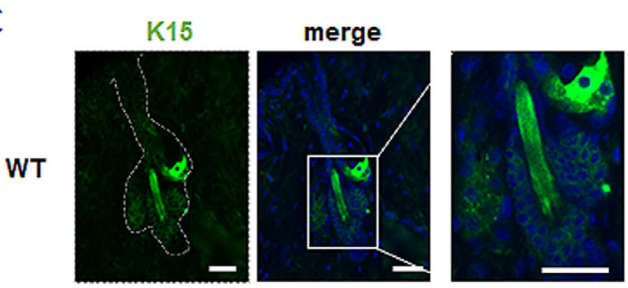

Cd133
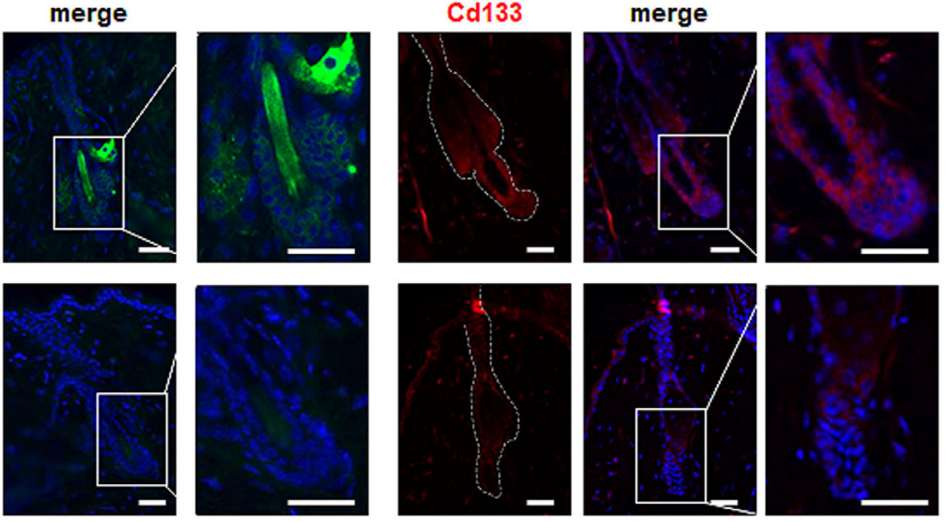
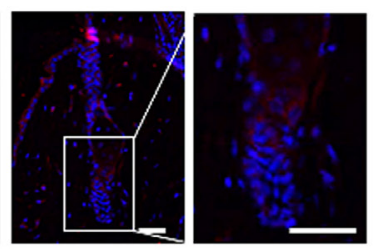

$\mathrm{Bar}=20 \mu \mathrm{m}$

Fig. 5. Fpr2 ablation blocks activation of HFSCs and DPCs. (A) Western blot analysis and (B) cumulative densitometric analysis for K15 and Cd133 in dorsal skin of WT and Fpr2 KO mice. Band densities were normalized to the expression level of $\beta$-actin, an internal control. Data represent the mean \pm SEM (unpaired two-sample student's $t$-test; $n \geq 3$ /group, * $p<0.05$ vs WT). (C) Representative images of immunofluorescence staining for K15 (green) and Cd133 (red) in the dorsal skin sections from these mice $(\times 40)$. Magnified images $(\times 100)$ are shown in right panel. DAPI was used in staining for nucleus. The white dashed lines indicate the HF regions (Scale bars: $20 \mu \mathrm{m}$ ). DPC, dermal papilla cells; HFSC, hair follicle stem cells. K15, keratin 15. 
the mechanism underlying hair loss, it remains a poorly understood condition. This limited understanding of hair loss hinders the development of applicable therapies for all types of hair loss. Therefore, it is necessary to discover novel mediators of hair loss. This study revealed the effect of FPR2 on hair regeneration using an Fpr2 KO mice model. Fpr2 KO mice displayed postnatal hair loss, and we observed obvious histological differences between the WT and Fpr2 KO mice. A thickened hypodermal adipocyte layer and dystrophic HFs with distorted shapes, cyst-like structures, and excessive accumulations of epithelial cells were apparent in the Fpr2 KO mice. To determine the role of Fpr2 in this HF impairment, we first examined Fpr2 expression in the dorsal skin tissue of mice, and revealed that Fpr2 was expressed by dermal fibroblasts in the outer layer of the surrounding lower parts in the HF. Dermal fibroblasts play a critical role in skin structure and integrity by synthesizing the extracellular matrix that supports the skin structure (Thangapazham et al., 2014). In addition, dermal fibroblasts were shown to have an inductive effect on HF neogenesis by promoting the activity of DPCs and HFSCs (le Riche et al., 2019). Given the actions of dermal fibroblasts in inducing hair regeneration, FPR2 expression in dermal fibroblasts suggests that FPR2 has the potential to regulate hair regeneration by influencing the activation of DPCs and HFSCs.

Hair regeneration is mediated by a cycle of structural changes (Stenn \& Paus, 2001; Schneider et al., 2009). Hence, the activation and stability of the hair regeneration cycle are key factors in achieving an intact HF. However, HF loss is often accompanied by the termination and/or dysregulation of the hair regeneration cycle, such as a shortened anagen phase or a failed transition from the telogen to anagen phase (Ji et al., 2021). The activity of HFSCs and DPCs is the main driving force for the continuous hair regeneration cycle and is regulated by various signaling molecules (Rahmani et al., 2014; Chen et al., 2020a). Among these signaling molecules, SHH, $\mathrm{Wnt} / \beta$-catenin, and BMP are the most prominent in hair regeneration (Rishikaysh et al., 2014). SHH accelerates HF growth by inducing the activation of HFSCs and DPCs (St-Jacques et al., 1998; Lim et al., 2018). After the anagen phase is initiated, the increased production of $\mathrm{SHH}$ promotes HFSC proliferation and DPC maturation (St-Jacques et al., 1998; Lim et al., 2018). Cui et al. reported that skin-specific Shh-depleted mice had total hair loss over their entire bodies (Cui et al., 2011). Ubiquitous Shh KO mice were unable to develop HFs during development despite having hair germs (Chiang et al., 1999). It was also shown that the administration of recombinant human SHH protein increased hair density and hair length in mice (Yu et al., 2019). Shh stimulates hair regeneration by modulating Wnt/ $\beta$-catenin (Avigad Laron et al., 2018). Laron et al. revealed that Shh signaling in the DPCs fine-tunes the activation of Wnt/ $\beta$-catenin signaling that results in the proliferation of HFSCs for hair regeneration (Avigad Laron et al., 2018). Wnt/ $\beta$-catenin signaling is also implicated in the development of skin, hair, and related appendages (Millar et al., 1999). $\beta$-catenin is known to be essential for HFSC activation, via its collaboration with Lymphoid enhancer-binding factor-1 (LEF-1), and it controls their growth (van Genderen et al., 1994; Huelsken et al., 2001). Huelsken et al. demonstrated that $\beta$-catenin deletion led to subsequent hair loss and small cystic HFs after normal morphogenesis (Huelsken et al., 2001). In line with these findings, we found that the levels of Shh and $\beta$-catenin in HFs were remarkably lower in Fpr2 KO mice with extensive hair loss and small cystic HFs than WT mice (Fig. 1-3). In addition, expression of Bmp2 and Bmp4, which block anagen induction, increased in Fpr2 KO mice compared with WT mice (Fig. 4). Plikus et al. reported that Bmp2 and Bmp4 were produced by extra-follicular sources (Plikus et al., 2008). Given that most BMP2 is produced by hypodermis adipocytes, the thickened hypodermis adipocyte layer in the Fpr2 $\mathrm{KO}$ mice seems to be related to the increased expression of Bmp2 (Plikus et al., 2008).

In conclusion, we have demonstrated that the signaling molecules regulating the activity of HFSCs and DPCs are dysregulated in Fpr2 KO mice, and that hair regeneration is impaired, 
resulting in hair loss after morphogenesis. Although the mechanism by which FPR2 interacts with the signaling molecules in pathogenic hair loss remains unclear, our results indicate that FPR2 is a key modulator of HFs and has therapeutic potential for the prevention and/or treatment of hair loss.

\section{REFERENCES}

Abaci HE, Coffman A, Doucet Y, Chen J, Jacków J, Wang E, Guo Z, Shin JU, Jahoda CA, Christiano AM (2018) Tissue engineering of human hair follicles using a biomimetic developmental approach. Nat Commun 9:5301.

Avigad Laron E, Aamar E, Enshell-Seijffers D (2018) The mesenchymal niche of the hair follicle induces regeneration by releasing primed progenitors from inhibitory effects of quiescent stem cells. Cell Rep 24:909-921.

Bose A, Teh MT, Mackenzie IC, Waseem A (2013) Keratin k15 as a biomarker of epidermal stem cells. Int J Mol Sci 14:19385-19398.

Botchkarev VA, Botchkareva NV, Sharov AA, Funa K, Huber O, Gilchrest BA (2002) Modulation of BMP signaling by noggin is required for induction of the secondary (nontylotrich) hair follicles.J Invest Dermatol 118:3-10.

Carreño-Orellana N, Moll-Manzur C, Carrasco-Zuber JE, Álvarez-Véliz S, Berroeta-Mauriziano D, Porras-Kusmanic N (2016) Finasteride adverse effects: An update. Rev Med Chil 144:15841590.

Chen CL, Huang WY, Wang EHC, Tai KY, Lin SJ (2020a) Functional complexity of hair follicle stem cell niche and therapeutic targeting of niche dysfunction for hair regeneration. J Biomed Sci 27:43.

Chen T, Xiong M, Zong X, Ge Y, Zhang H, Wang M, Won Han G, Yi C, Ma L, Ye RD, Xu Y, Zhao Q,Wu B (2020b) Structural basis of ligand binding modes at the human formyl peptide receptor 2. Nat Commun 11:1208.

Chiang C, Swan RZ, Grachtchouk M, Bolinger M, Litingtung Y, Robertson EK, Cooper MK, Gaffield W, Westphal H, Beachy PA, Dlugosz AA (1999) Essential role for Sonic hedgehog during hair follicle morphogenesis. Dev Biol 205:1-9.

Cui CY, Kunisada M, Childress V, Michel M, Schlessinger D (2011) Shh is required for Tabby hair follicle development. Cell Cycle 10:3379-3386.

Fang F, Ooka K, Sun X, Shah R, Bhattacharyya S, Wei J, Varga J (2013) A synthetic TLR3 ligand mitigates profibrotic fibroblast responses by inducing autocrine IFN signaling. J Immunol 191:2956-2966.

Gaudin A, Tolar M, Peters OA (2018) Lipoxin $\mathrm{A}_{4}$ attenuates the inflammatory response in stem cells of the apical papilla via ALX/FPR2. Sci Rep 8:8921.

Genander M, Cook PJ, Ramsköld D, Keyes BE, Mertz AF, Sandberg R, Fuchs E (2014) BMP signaling and its $\mathrm{pSMAD} 1 / 5$ target genes differentially regulate hair follicle stem cell lineages. Cell Stem Cell 15:619-633.

Grimalt R (2005) Psychological aspects of hair disease.J Cosmet Dermatol 4:142-147.

Huelsken J, Vogel R, Erdmann B, Cotsarelis G, Birchmeier W (2001) $\beta$-Catenin controls hair follicle morphogenesis and stem cell differentiation in the skin. Cell 105:533-545.

Ito Y, Hamazaki TS, Ohnuma K, Tamaki K, Asashima M, Okochi H (2007) Isolation of murine hair-inducing cells using the cell surface marker prominin-1/CD133. J Invest Dermatol 127:1052-1060. 
Ji S, Zhu Z, Sun X, Fu X (2021) Functional hair follicle regeneration: An updated review. Signal Transduct Target Ther 6:66.

Krieger K, Millar SE, Mikuda N, Krahn I, Kloepper JE, Bertolini M, Scheidereit C, Paus R, Schmidt-Ullich R (2018) NF- $\kappa$ B participates in mouse hair cycle control and plays distinct roles in the various pelage hair follicle types.J Invest Dermatol 138:256-264.

le Riche A, Aberdam E, Marchand L, Frank E, Jahoda C, Petit I, Bordes S, Closs B, Aberdam D (2019) Extracellular vesicles from activated dermal fibroblasts stimulate hair follicle growth through dermal papilla-secreted norrin. Stem Cells 37:1166-1175.

Lee SW, Juhasz M, Mobasher P, Ekelem C, Mesinkovska NA (2018) A systematic review of topical finasteride in the treatment of androgenetic alopecia in men and women. J Drugs Dermatol 17:457-463.

Lim CH, Sun Q, Ratti K, Lee SH, Zheng Y, Takeo M, Lee W, Rabbani P, Plikus MV, Cain JE, Wang DH, Watkins DN, Millar S, Taketo MM, Myung P, Cotsarelis G, Ito M (2018) Hedgehog stimulates hair follicle neogenesis by creating inductive dermis during murine skin wound healing. Nat Commun 9:4903.

Millar SE, Willert K, Salinas PC, Roelink H, Nusse R, Sussman DJ, Barsh GS (1999) WNT signaling in the control of hair growth and structure. Dev Biol 207:133-149.

Müller-Röver S, Handjiski B, van der Veen C, Eichmüller S, Foitzik K, McKay IA, Stenn KS, Paus R (2001) A comprehensive guide for the accurate classification of murine hair follicles in distinct hair cycle stages. J Invest Dermatol 117:3-15.

Plikus MV, Mayer JA, de la Cruz D, Baker RE, Maini PK, Maxson R, Chuong CM (2008) Cyclic dermal BMP signalling regulates stem cell activation during hair regeneration. Nature 451:340344.

Plikus MV, Widelitz RB, Maxson R, Chuong CM (2009) Analyses of regenerative wave patterns in adult hair follicle populations reveal macro-environmental regulation of stem cell activity. Int J Dev Biol 53:857-868.

Rahmani W, Abbasi S, Hagner A, Raharjo E, Kumar R, Hotta A, Magness S, Metzger D, Biernaskie J (2014) Hair follicle dermal stem cells regenerate the dermal sheath, repopulate the dermal papilla, and modulate hair type. Dev Cell 31:543-558.

Rishikaysh P, Dev K, Diaz D, Qureshi WMS, Filip S, Mokry J (2014) Signaling involved in hair follicle morphogenesis and development. Int J Mol Sci 15:1647-1670.

Rossi A, Cantisani C, Melis L, Iorio A, Scali E, Calvieri S (2012) Minoxidil use in dermatology, side effects and recent patents. Recent Pat Inflamm Allergy Drug Discov 6:130-136.

Schneider MR, Schmidt-Ullrich R, Paus R (2009) The hair follicle as a dynamic miniorgan. Curr Biol 19:R132-R142.

Seale LR, Eglini AN, McMichael AJ (2016) Side effects related to $5 \alpha$-reductase inhibitor treatment of hair loss in women: A review. J Drugs Dermatol 15:414-419.

St-Jacques B, Dassule HR, Karavanova I, Botchkarev VA, Li J, Danielian PS, McMahon JA, Lewis PM, Paus R, McMahon AP (1998) Sonic hedgehog signaling is essential for hair development. Curr Biol 8:1058-1068.

Stenn KS, Paus R (2001) Controls of hair follicle cycling. Physiol Rev 81:449-494.

Strazzulla LC, Wang EHC, Avila L, Lo Sicco K, Brinster N, Christiano AM, Shapiro J (2018) Alopecia areata: Disease characteristics, clinical evaluation, and new perspectives on pathogenesis.J Am Acad Dermatol 78:1-12.

Suchonwanit P, Thammarucha S, Leerunyakul K (2019) Minoxidil and its use in hair disorders: A review. Drug Des Devel Ther 13:2777-2786.

Thangapazham RL, Darling TN, Meyerle J (2014) Alteration of skin properties with autologous 
dermal fibroblasts. Int J Mol Sci 15:8407-8427.

Tsuruki T, Yoshikawa M (2006) Orally administered FPRL1 receptor agonist peptide MMK1 inhibits etoposide-induced alopecia by a mechanism different from intraperitoneally administered MMK-1. Peptides 27:820-825.

van Genderen C, Okamura RM, Fariñas I, Quo RG, Parslow TG, Bruhn L, Grosschedl R (1994) Development of several organs that require inductive epithelial-mesenchymal interactions is impaired in LEF-1-deficient mice. Genes Dev 8:2691-2703.

VanCompernolle SE, Clark KL, Rummel KA, Todd SC (2003) Expression and function of formyl peptide receptors on human fibroblast cells. J Immunol 171:2050-2056.

Yu SH, Kim Y, Jung N, Hwang JW, Kim N, Ha JC, Kim MJ, Lee Y, Choi YS, Han K, Kim CH (2019) Hair growth-promoting effect of recombinant human sonic hedgehog proteins. Biomed Dermatol 3:7.

Zhang J, He XC, Tong WG, Johnson T, Wiedemann LM, Mishina Y, Feng JQ Li L (2006) Bone morphogenetic protein signaling inhibits hair follicle anagen induction by restricting epithelial stem/progenitor cell activation and expansion. Stem Cells 24:2826-2839.

Zhang L, Wang G, Chen X, Xue X, Guo Q, Liu M, Zhao J (2017) Formyl peptide receptors promotes neural differentiation in mouse neural stem cells by ROS generation and regulation of PI3K-AKT signaling. Sci Rep 7:206. 
